Pathologic basis of disease. R.S. Cotran, V. Kumar, and S.L. Robbins, editors. W.B. Saunders. Philadelphia, Pennsylvania, USA. 51-92.

18. Maiese, K., Li, F., and Chong, Z.Z. 2005. New avenues of exploration for erythropoietin. JAMA. 293:90-95.

19. Chong, Z.Z., and Maiese, K. 2007. Erythropoietin involves the phosphatidylinositol 3-kinase pathway, 14-3-3 protein and FOXO3a nuclear trafficking to preserve endothelial cell integrity. Br. J. Phar- macol. 150:839-850.

20. Fliser, D., Bahlmann, F.H., and Haller, H. 2006. EPO: renoprotection beyond anemia correction. Pediatr. Nephrol. 21:1785-1789.

21. Ferrara, N. 2001. Role of vascular endothelial growth factor in regulation of physiological angiogenesis. Am. J. Physiol. Cell Physiol. 280:C1358-C1366.

22. Reinders, M.E., et al. 2003. Proinflammatory functions of vascular endothelial growth factor in alloimmunity. J. Clin. Invest. 112:1655-1665.
23. Denton, M.D., et al. 2004. TNP-470, an angiogenesis inhibitor, attenuates the development of allograft vasculopathy. Transplantation. 78:1218-1221.

24. Contreras, A.G., Flynn, E., Meyer, E., Sho, M., and Briscoe, D.M. 2007. Anti-angiogenesis therapy interrupts the progression of allograft vasculopathy [abstract]. Am. J. Transplant. 7:331.

25. Lemstrom, K.B., et al. 2002. Vascular endothelial growth factor enhances cardiac allograft arteriosclerosis. Circulation. 105:2524-2530.

\title{
Staying awake puts pressure on brain arousal systems
}

\author{
Giulio Tononi and Chiara Cirelli
}

Department of Psychiatry, University of Wisconsin-Madison, Madison, Wisconsin, USA.

\begin{abstract}
Many brain centers are involved in keeping us awake. One example is the recently discovered hypocretin system located in the posterior hypothalamus. In this issue of the JCI, Rao et al. show that, in mice, synapses targeting hypocretin neurons become stronger when wakefulness is prolonged beyond its physiological duration (see the related article beginning on page 4022). This increase in synaptic strength may be one of the mechanisms that help us to stay awake when we are sleep deprived, but it may also represent one of the signals telling the brain that it is time to sleep.
\end{abstract}

Sleep appears to be a universal phenomenon, since it is present in all species that have been carefully studied so far (1). A lot is known about the brain areas responsible for promoting arousal and the maintenance of wakefulness, as well as those areas crucial for the initiation and maintenance of sleep. The functions of sleep, on the other hand, remain unclear, although it is clear that if wakefulness is prolonged beyond its physiological duration (approximately 16 hours in humans), severe physiological and cognitive deficits occur (2).

\section{What keeps us awake?}

There are several neurochemical systems with diffuse projections that promote and maintain wakefulness. These include the noradrenergic cells in the locus coeruleus (LC); cholinergic cells in the pedunculopontine tegmental nucleus (PPTN), lat-

Nonstandard abbreviations used: LC, locus coeruleus; LDTN, lateral dorsal tegmental nucleus; LTP, long-term potentiation; mEPSC, miniature excitatory postsynaptic current; NREM, non-rapid eye movement; PPTN, pedunculopontine tegmental nucleus; TMN, tuberomamillary nucleus; VTA, ventral tegmental area. Conflict of interest: Giulio Tononi has served as a consultant to Tikvah Therapeutics Inc. Chiara Cirelli has declared that no conflict of interest exists.

Citation for this article: J. Clin. Invest. 117:3648-3650 (2007). doi:10.1172/JCI34250. eral dorsal tegmental nucleus (LDTN), and basal forebrain; histaminergic cells in the tuberomamillary nucleus (TMN) of the posterior hypothalamus; and glutamatergic neurons in various structures in the central nervous system (3). These cells fire at a higher rate during waking than during non-rapid eye movement (NREM) sleep. Dopamine-containing neurons in the substantia nigra and ventral tegmental area (VTA) also modulate arousal. VTA cells fire tonically during quiet wakefulness and NREM sleep, and in bursts (inducing massive release of dopamine) during appetitive waking behavior and REM sleep (4). Psychostimulants, such as amphetamines and cocaine, that block the reuptake of monoamines including norepinephrine, dopamine, and serotonin promote prolonged wakefulness and increase both cortical activation and behavioral arousal (5). The most recent wakefulness-promoting system to be discovered is the hypocretin system. The peptide hypocretin (also known as orexin) is produced by cells in the posterior hypothalamus that provide excitatory input to all remaining wakefulnesspromoting areas, including the LC, PPTN, LDTN, VTA, basal forebrain, and TMN (6) (Figure 1). Hypocretin neurons are most active during waking, especially in relation to motor activity and exploratory behavior, and almost stop firing during both NREM and REM sleep.

Wakefulness-promoting systems appear to be redundant to some extent, which may explain why specific lesions that affect one or a few of these brain areas do not result in a complete and permanent suppression of cortical activation and wakefulness (7). A common mechanism through which these systems produce cortical activation is by closing potassium channels on the cell membrane of cortical and thalamic neurons, thus keeping cells depolarized and ready to fire (8). At sleep onset, wakefulness-promoting neuronal groups are actively inhibited by antagonistic neuronal populations located in the hypothalamus $(9,10)$ and the basal forebrain $(11,12)$, most of which are GABAergic (transmit or secrete $\gamma$-aminobutyric acid). Exactly how the switch from wakefulness to sleep occurs, however, remains unclear.

\section{The homeostatic regulation of sleep}

What is clear is that sleep is regulated through both circadian and homeostatic mechanisms (13). The circadian regulation is responsible for the change in sleep propensity depending on the time of day, with obvious adaptive advantages. Humans, for instance, are a diurnal species (active during the daytime and sleeping mainly at night), while mice and rats are nocturnal and sleep mainly during the day. The homeostatic regulation of sleep, on the other hand, is responsible for the fact that the longer one stays awake, the stronger the pressure to go to sleep becomes (sleepiness increases). Also, the subsequent sleep is 


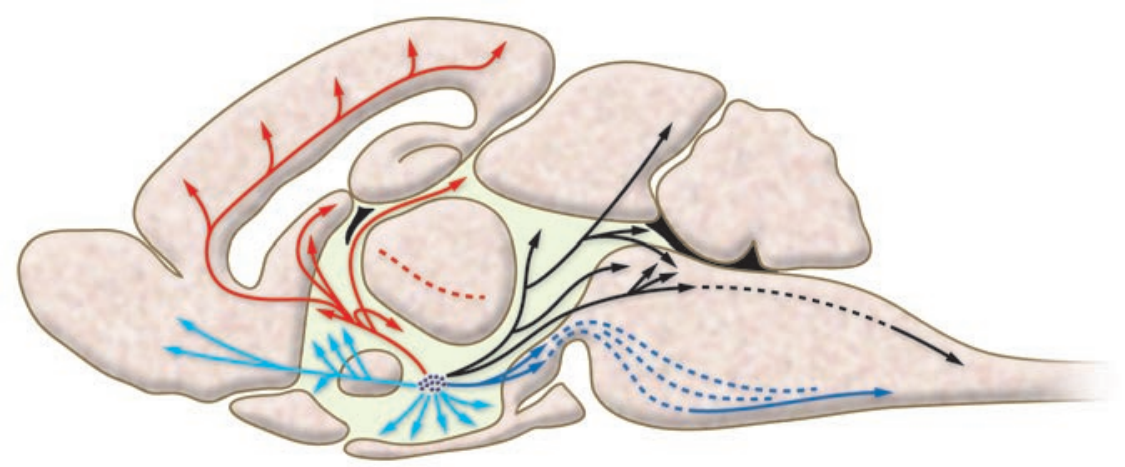

Figure 1

The hypocretin system in the posterior hypothalamus (dots) and its projections (arrows) to most brain regions. Hypocretin provides excitatory input to other wakefulness-promoting areas. Hypocretin-labeled neurons are shown in purple, the dorsal ascending pathway in red, the ventral ascending pathway in light blue, the dorsal descending pathway in black, and the ventral descending pathway in dark blue. Image reproduced with permission from Trends in Neurosciences (21).

longer and more consolidated (with fewer brief awakenings). Influential models of sleep regulation (13) have suggested that the homeostatic component predicts the existence in the brain of a distinct physiological/biochemical/molecular process that builds up during wakefulness and declines during sleep, but the nature of such a process is still unclear.

\section{Synaptic potentiation in hypocretin neurons}

In this issue of the JCI, Rao et al. (14) have made an important contribution to our understanding of how the activity of the hypocretin wakefulness-promoting system changes during prolonged wakefulness. The authors postponed, for 2-4 hours, the normal sleep period in mice either by injecting the animals with the wake-promoting drug modafinil or by sleep depriving them using gentle handling. They then sacrificed the mice to measure miniature excitatory postsynaptic currents (mEPSCs) in brain slices and found that this increase in waking duration led to long-term potentiation (LTP; a long-lasting improvement in the communication between 2 neurons, via their synapses, that usually results from simultaneous neuronal stimulation) of glutamatergic synapses on identified hypocretin neurons. Both the frequency and the amplitude of mEPSCs were increased, suggesting changes in both the pre- and postsynaptic components of synaptic transmission. Pretreatment with a selective D1 antagonist, SCH23390, blocked the modafinil-induced increase in mEPSC frequency, but not in amplitude, indicating that dopaminergic transmission is involved in the potentiation of the presynaptic component, while other neuromodulators, most likely norepinephrine and histamine, may play a role in the potentiation of the postsynaptic component. By measuring evoked EPSCs in the same hypocretin neurons, the authors also found that the contribution to EPSCs of glutamatergic $\alpha$ amino-3-hydroxy-5-methyl-4-isoxazolepropionic acid (AMPA) receptors was increased relative to that of $N$-methyl-D-aspartic acid receptors. Moreover, in mice chronically treated with modafinil (once daily for 1 week) the authors found not only an increase in frequency and amplitude of mEPSCs, but also an increase in the number of asymmetric synapses on hypocretin cells. Finally, Rao et al. show that the LTPlike changes observed 2 hours after acute modafinil treatment partially occlude the ability of forskolin, a drug that increases the intracellular levels of cyclic AMP and normally induces synaptic potentiation (the long-lasting strengthening of the response of a neuron after it has received a message from another neuron across the synapse), to further potentiate synapses. Thus, this elegant and comprehensive study shows that even a few hours of wakefulness trigger synaptic potentiation in one of the wakefulness-promoting systems, suggesting that hypocretin cells can somehow sense how long the brain has been awake. The authors speculate that the increase in synaptic strength in these neurons may be at least one of the mechanisms by which prolonged wakefulness is maintained despite an increase in sleep pressure.

\section{Future directions}

In future studies, other wakefulness-promoting cells - for instance, those present in the LC, PPTN, or TMN - should be examined. It could be that wakefulnessinduced synaptic potentiation may take place in other wakefulness-promoting systems. If so, synaptic changes in the input to neuromodulatory systems could represent one of several possible mechanisms underlying the homeostatic regulation of sleep. Obviously, future causal experiments are needed to address these questions. Another important result shown by Rao et al. (14) is the partial occlusion of LTP observed after acute modafinil treatment. This clearly suggests that the ability of glutamatergic synapses on hypocretin cells to undergo synaptic potentiation in vivo can become saturated. How quickly and easily can this happen? Intriguingly, in vitro studies in the hippocampus have shown that prolonged waking impairs the induction of LTP but favors the induction of long-term depression (e.g., refs. 15-17). Moreover, in both cerebral cortex and hippocampus, learning during wakefulness can strengthen synapses to near the maximum of their modification range, impairing the further induction of LTP $(18,19)$. These experiments suggest that wakefulness-induced synaptic potentiation may be a more widespread phenomenon that could extend beyond neuromodulatory systems to some of their targets in the brain (20). Finally, the results of Rao et al. raise an intriguing question: if wakefulness is associated with the progressive potentiation of synapses on hypocretin neurons, there must be a mechanism by which a synaptic balance is maintained - obviously, synapses cannot simply keep strengthening indefinitely. The most straightforward possibility, of course, would be that such balance is achieved in subsequent sleep periods. Thanks to this provocative study, we will probably know the answer soon.

\section{Acknowledgments}

This work was partially supported by an NIH Director's Pioneer Award to Giulio Tononi.

Address correspondence to: Chiara Cirelli or Giulio Tononi, Department of Psychiatry, University of Wisconsin-Madison, 6001 Research Park Blvd., Madison, Wisconsin 53719, USA. Phone: (608) 263-6063; Fax: (608) 265 2953; E-mail: ccirelli@wisc.edu (C. Cirelli); gtononi@wisc.edu (G. Tononi). 
1. Tobler, I. 2005. Phylogeny of sleep regulation. In Principles and practice of sleep medicine. M.H. Kryger, T. Roth, and W.C. Dement, editors. 4th edition. W.B. Saunders. Philadelphia, Pennsylvania, USA. 77-90.

2. Durmer, J.S., and Dinges, D.F. 2005. Neurocognitive consequences of sleep deprivation. Semin. New rol. 25:117-129.

3. Jones, B.E. 2005. From waking to sleeping: neuronal and chemical substrates. Trends Pharmacol. Sci. 26:578-586

4. Dahan, L., et al. 2007. Prominent burst firing of dopaminergic neurons in the ventral tegmental area during paradoxical sleep. Neuropsychopharmacology. 32:1232-1241.

5. Monti, J.M., and Monti, D. 2007. The involvement of dopamine in the modulation of sleep and waking. Sleep Med. Rev. 11:113-133.

6. Sakurai, T. 2007. The neural circuit of orexin (hypocretin): maintaining sleep and wakefulness. Nat. Rev. Neurosci. 8:171-181.

7. Denoyer, M., Sallanon, M., Buda, C., Kitahama, K., and Jouvet, M. 1991. Neurotoxic lesion of the mesencephalic reticular formation and/or the posterior hypothalamus does not alter waking in the cat. Brain Res. 539:287-303.

8. McCormick, D.A., and Bal, T. 1997. Sleep and arousal: thalamocortical mechanisms. Ann. Rev. Neurosci. 20:185-215.

9. Sherin, J.E., Shiromani, P.J., McCarley, R.W., and Saper, C.B. 1996. Activation of ventrolateral preoptic neurons during sleep. Science. 271:216-219.

10. Suntsova, N., Szymusiak, R., Alam, M.N., Guzman-Marin, R., and McGinty, D. 2002. Sleep-waking discharge patterns of median preoptic nucleus neurons in rats. J. Physiol. 543:665-677.

11. Detari, L., Rasmusson, D.D., and Semba, K. 1997. Phasic relationship between the activity of basal forebrain neurons and cortical EEG in urethaneanesthetized rat. Brain Res. 759:112-121.

12. Lee, M.G., Manns, I.D., Alonso, A., and Jones, B.E. 2004. Sleep-wake related discharge properties of basal forebrain neurons recorded with micropipettes in head-fixed rats. J. Neurophysiol. 92:1182-1198.

13. Borbély, A.A. 1982. A two process model of sleep regulation. Human Neurobiol. 1:195-204.

14. Rao, Y., et al. 2007. Prolonged wakefulness induces experience-dependent synaptic plasticity in mouse hypocretin/orexin neurons. J. Clin. Invest.
117:4022-4033.

15. McDermott, C.M., et al. 2003. Sleep deprivation causes behavioral, synaptic, and membrane excitability alterations in hippocampal neurons. J. Neurosci. 23:9687-9695.

16. Kopp, C., Longordo, F., Nicholson, J.R., and Luthi, A. 2006. Insufficient sleep reversibly alters bidirectional synaptic plasticity and NMDA receptor function. J. Neurosci. 26:12456-12465.

17. Tartar, J.L., et al. 2006. Hippocampal synaptic plasticity and spatial learning are impaired in a rat model of sleep fragmentation. Eur. J. Neurosci. 23:2739-2748.

18. Rioult-Pedotti, M.S., Friedman, D., and Donoghue, J.P. 2000. Learning-induced LTP in neocortex. Science. 290:533-536.

19. Whitlock, J.R., Heynen, A.J., Shuler, M.G., and Bear, M.F. 2006. Learning induces long-term potentiation in the hippocampus. Science. 313:1093-1097.

20. Tononi, G., and Cirelli, C. 2006. Sleep function and synaptic homeostasis. Sleep Med. Rev. 10:49-62.

21. Kilduff, T.S., and Peyron, C. 2000. The hypocretin/ orexin ligand-receptor system: implications for sleep and sleep disorders. Trends Neurosci. 23:359-365.

\title{
Structural reengineering of imatinib to decrease cardiac risk in cancer therapy
}

\author{
George D. Demetri
}

Ludwig Center for Cancer Research, Dana-Farber Cancer Institute and Harvard Medical School, Boston, Massachusetts, USA. Clinical and Translational Research, Ludwig Institute for Cancer Research, New York, New York, USA, and Zurich, Switzerland.

Imatinib, a selective, small-molecule tyrosine inhibitor, has life-saving clinical activity in certain cancers, but questions have been raised about the potential for cardiac toxicity through inhibition of its target, $A B L$ kinase. In this issue of the JCI, Fernández et al. describe a novel method by which the ABL-inhibitory activity of imatinib was deleted by modifying its chemical structure (see the related article beginning on page 4044). The anticancer activity of the reengineered agent, called $\mathrm{WBZ}_{-} 4$, was instead preserved against gastrointestinal stromal tumors in both in vitro and in vivo models via inhibition of KIT tyrosine kinase, and the desired safety was demonstrated with less cardiotoxicity of $\mathrm{WBZ}_{4} 4$ compared with imatinib via the inhibition of JNK. The study shows that structural reengineering of a kinaseinhibitory drug to improve tolerability while preserving efficacy is feasible.

\section{Signal transduction inhibitors in cancer therapy}

The molecular genetic basis for many forms of cancer has been elucidated; the etiologic connection between mutational activation of certain oncogenic gene products and

Nonstandard abbreviations used: CML, chronic myelogenous leukemia; GIST, gastrointestinal stromal tumor.

Conflict of interest: The author receives research support from Ariad Pharmaceuticals Inc., Bristol-Myers Squibb Co., Daiichi Sankyo Inc., Infinity Pharmaceuticals, Johnson \& Johnson, Novartis Pharmaceuticals Corp., and Pfizer Inc. and consultant fees from Ariad Pharmaceuticals Inc. and Ziopharm Oncology Inc.

Citation for this article: J. Clin. Invest. 117:3650-3653 (2007). doi:10.1172/JCI34252. human cancer are now fully evident for certain diseases such as chronic myelogenous leukemia (CML) and gastrointestinal stromal tumors (GISTs), in which single mutations lead to uncontrolled tyrosine kinase activity of the BCR-ABL fusion kinase and the KIT kinase, respectively. The concept of using rationally designed small molecules to inhibit such oncogenic kinase signaling to treat cancer had its roots in the work of Alexander Levitzki and others in the 1990s (1). However, the successful implementation of this concept came with the dramatic success of imatinib mesylate (now known as Gleevec in the United States and Glivec in the rest of the world), which has revolutionized the treatment of patients with CML. A multi-institutional collaboration between Brian Druker and Nicolas Lyden and their colleagues with pharma led to the focused testing of imatinib to target BCR-ABL kinase activity $(2,3)$, and subsequent multi-institutional collaborations targeted the KIT kinase in GISTs as another therapeutic application $(2,4)$. The result of this work has given hope to the field of oncology, validating the concept that both hematologic malignancies and solid tumors in humans can be treated successfully with agents that target elements of the molecular pathogenesis of the disease. Both CML and GISTs were ideal proof-of-concept diseases, because single genetic mutations play a disproportionately large role in the pathobiology of these diseases.

\section{Clinical impact of selective or multitargeted kinase inhibitors in cancer treatment}

Imatinib has become the "poster child" drug of small-molecule molecular therapeutics, with a spectrum of activity that is reasonably selective for certain cell signaling pathways. It is important to note that 